\section{Digital Imaging for TEM Part 1}

Anthony D. Buonaquisit, Advanced Microscopy Techniques

We are all familiar with digital imaging for SEM instruments. Digital imaging for TEM applications is not as well established. Nevertheless, it seems clear that it will not be long before digital imaging for TEM becomes common place. Systems are improving and costs are plummeting. With this in mind it is timely to review what digital imaging for TEM involves.

In normal TEM operation an electron bream is scattered through a thin section of a sample. Physical mechanisms cause the electrons of the beam to scatter, producing bright-field images, dark-field images and diffraction patterns. The operator adjusts the instrument to display one of these images on the instrument's viewing screen. A photographic record is collected by flipping the viewing screen and exposing a sheet of film held in the TEM's camera. Exposed negatives can be removed for developing and printing in batches, using standard darkroom techniques.

Digital imaging systems (Figure 1) consist of a scintillator screen, a light sensitive digital camera, a frame grabber computer card and a computer system.

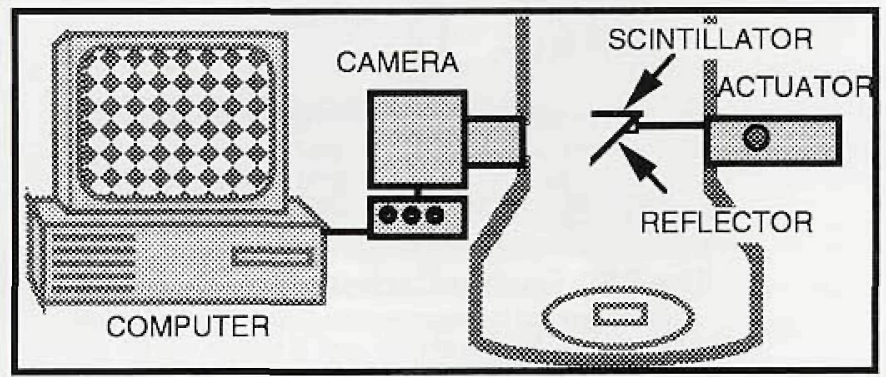

Figure 1: Side Mount TEM Digital Imaging System

The scintillator converts the electron image into a photon image. Scintillators can be produced from phosphor powder or single crystals. Both materials have found application though the phosphor powders are said to offer the better sensitivity, especially for low dose imaging.

The digital camera is coupled to the scintillator using either a fiber optic bundle or a high quality lens system. The former provides more efficient light collection. The latter allows a much wider field of view and potentially higher resolution

Modern digital cameras are designed around a charge coupled device (CCD). The CCD sensor is an integrated circuit (Figure 2) that is fabricated to form a two dimensional array of light sensitive cells. When a photon penetrates an individual cell it produces a small charge which is held within the cell. The amount of charge held within a cell is proportional to the number of photons that penetrated the cell while it was exposed. On-chip circuitry "reads" the amount charge that has been stored in each cell, and thus records an image frame.

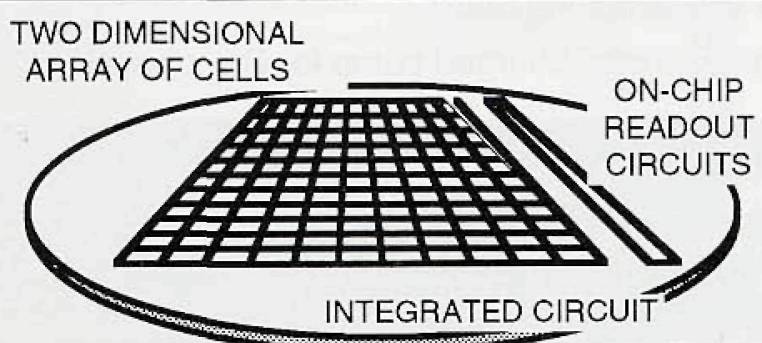

Figure 2: Schematic of CCD Chip Camera
$C C D$ arrays can have different formats producing square or rectangular active areas. A CCD array consisting of cells arranged in 384 rows and 512 columns would be relatively small and might be called a "500 line camera." A typical 100 line camera, might use 1317 rows and 1035 columns. Larger scale cameras such as 2000 line and 4000 line cameras are also available

The grey scale resolution of a CCD camera is determined by the electronics of it's readout circuity. Typical values are 256 gray levels, referred to as " 8 bits deep"; 1024 gray levels, referred to as "10 bits deep"; 4096 gray levels, referred to as " 12 bits deep"; and 16384 gray levels, referred to as " 14 bits deep".

The reading circuitry is fast but does take a finite time to read all the cells in the CCD array. It is therefore common practice to blank off the array during readout. This is achieved by either a mechanical shutter or beam blanking.

The exposure time between data readouts can be adjusted. Today's better systems operate at rates from 10 frames per second to many seconds per frame. Shorter exposure times are useful for focusing or "driving around" a sample Longer exposure times give best image recording. The duration of exposure is ultimately limited by the stability of the TEM and/or the sample.

Frame grabber cards are used to interface a standard PC or Macintosh with the camera system. They are the location of memory arrays referred to as "image planes", fast processing circuits and anything else the design engineer thinks might be useful for digital imaging. The idea is that computations using the frame grabber hardware will work faster than the the same thing done by software.

If the camera system and frame grabber board are designed to produce data in standard file formats, such as TIFF (Tagged Image File Format), the data are compatible with a wide world of commercial software applications programs Indeed all aspects of desktop publishing, desktop video and desktop networking are immediately open. In addition output devices can range from the simple laser printer to the latest dye sublimation or dry film processors.

Next time we will review the pro's and con's of digital imaging for TEM.

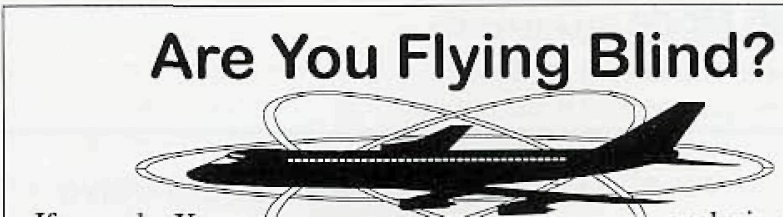

If you do $\mathrm{X}$-ray $(\mathrm{C}$ analysis on your SEM or TEM you need to know where the $\mathrm{X}$-rays are really coming from. Electron Flight Simulator ${ }^{\mathrm{TM}}$ is the first and only Windows based Monte Carlo software to give you the complete picture. With EFS you can see your X-ray generation volume for any sample chemistry at any tilt or KV. Model a bulk, film or film on a substrate. It is extremely easy to use, powerful and flexible. Without Electron Flight Simulator you are only flying blind.

Call or Fax for a free demo disk. (415) 345-8013

Small World P.O. Box 25284 San Mateo,CA 94402

\section{Digital Imaging For TEM}

AMT produces Digital Imaging Systems for all brands and models of TEM. Our international customer base makes us the market leader in 'Live Time' digital imaging for TEM. If you are thinking 'digital', call us! Why not visit our booth at MSA?

\section{Advanced Microscopy Techniques}

Box 661, Rowley MA 01969

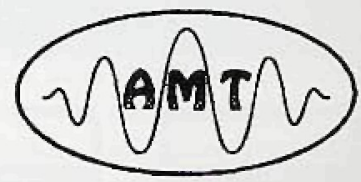

(508) 948-5507 


\section{The New Frontier In EDS \\ Is Decidedly Dry.}

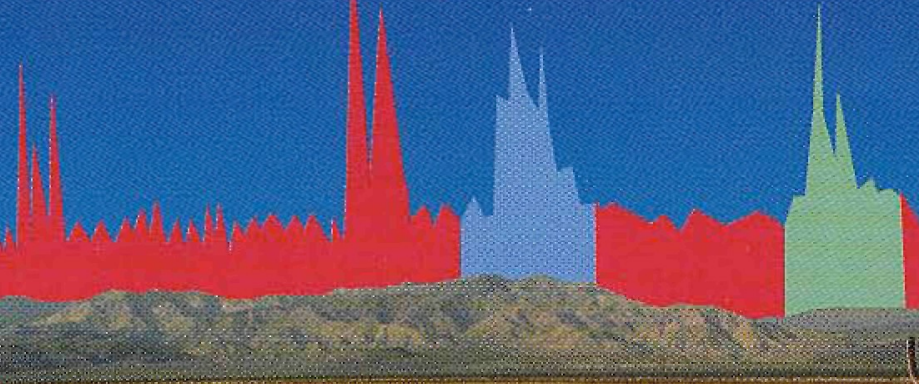

3 (

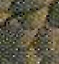
(1)

\section{Over 300 Scientists Have Moved to the Pioneering Dry Technology of Kevex.}

There's a new shape in energy dispersive $x$-ray detectors. One you'll be seeing a lot of... the unique Kevex SuperDry. The EDX detector that needs no liquid nitrogen... ever. Imagine freeing yourself from the ball-andchain of handling liquid nitrogen with no compromise in performance.

Hard data doesn't lie. We performed quantitative analyses using a $131 \mathrm{eV}$ resolution $\mathrm{LN}$ detector against a $149 \mathrm{eV}$ SuperDry on the same samples. Guess what? No difference in accuracy whatsoever, even for complex peak owerlaps. We repeated the experiment on high resolution digital $x$-ray maps... again, no difference at all in background levels. No wonder experienced users see right through the competition's clains.

Kevex is celebrating the success of our SuperDry detectors by introducing two new high performance models:

Quantum SuperDry Mark VI

$138 \mathrm{eV}$ resolution with boron detection guaranteed.
Quantum UltraDiy

A large $30 \mathrm{~mm}^{2}$ crystal for maximum count rates on difficult samples.

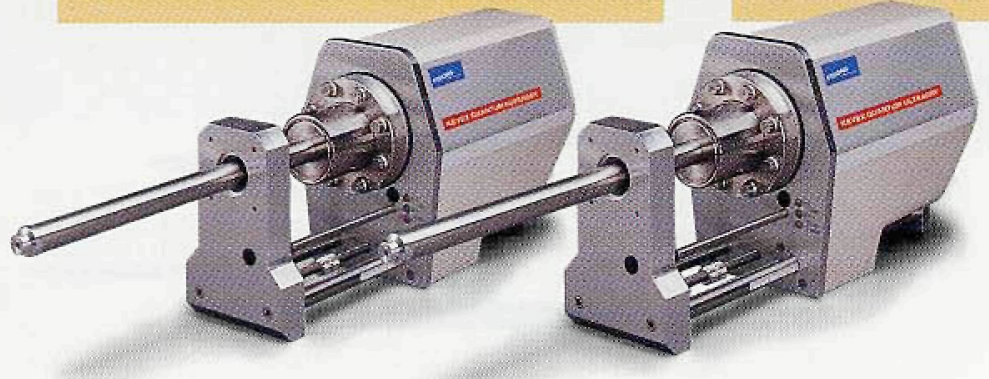

For applications proof and product information call 1-800-TO-KEVEX

\section{KEVEX}

\section{FISONS \\ Instruments}

Australia Homebush, Tel. (61) 2764-1155 Ganada Quebec, Tel. (514) 695-6257 Sweden Solna Tel. (08) 730-0295 Singapore Tel. (65) 760-8288

South Afica Kempton Park, Tel (11) $394-1410$ Spain Madid, Tel (01) 661-0642

United Kingdom Cheshive, Tel. (06) 06-86-1022 E Hong linstruments $\mathrm{Co}_{0}$

Taiwan Tel. (886) 2-755-2266

Fondis Electronic SA.

France, Tel. (01) $30-57-4747$

Fondis GmbH Germany, Tel. (49) 7251-716-182 Intesurface b.v

The Netherlands, Tel. (70) 414580

Micro Biological Lab, inc

Philipoines, Tel. (63) 2-597-876

Nissei Sangyo Co. Japan, Tel (81) 3-3504-7111 Rigong Intemational ho.

Korea, Tel. (82) 2-516-2686

Toshnival Bros. Pvi Ltd.

Indla, Tel (91) 22-22-1565

W. Pahisch SPA A ltaly, Tel (39) 2-353-73i

USA - Kevex World Headquarters, Valencia, CA, Tel (605) 295-0019, (800) TO KEVEX, $\operatorname{Fax}(605) 295-8714$ 